an involvement of DNA damage response pathway in nuclear reorganization during netotic initiation we selected an HL-60 based model. HL-60 is an acute myeloid leukemia cell line that preserved the capability of differentiation into granulocyte cell lineages. At first step, we performed differentiation of HL-60 cells into neutrophils with DMSO or ATRA. On the fifth day of differentiation, we have applied phorbol myristate to induce netotic cell death. Alternatively, we have treated granulocyte fraction isolated from bone marrow and blood of wild-type mice or from human donor blood with phorbol myristate. To study involvement of DNA damage response pathway we used an inhibitor of Wip1 phosphatase. Wip1 is a DNA damage induced nuclear phosphatase and a major negative switch of several DNA damage response proteins such as ATM, Chk1, Chk2, p53 and others. Results: Recently, several studies have suggested that nuclear structure of neutrophils plays an important role in their survival, their ability to migrate and control the release of NETs. First, we observed that Wip1 phosphatase expressed at high levels in the nucleus of differentiated HL-60 myeloid cells and in neutrophils. We observed that the inhibition of nuclear phosphatase Wip1 activity could extend mouse and human neutrophils and differentiated HL-60 cells survival through dephosphorylation and inhibition of its target proteins. Additionally, neutrophils treated with Wip1 inhibitors tends to show more segmented nuclei which have been correlated before with better anti-tumoral immune functions. Conclusions: In this context, we discovered that the inhibition of nuclear phosphatase Wip1 activity promotes neutrophil migration and control critical functions associated with nuclear reorganization during netotic type of cell death. The targeting of nuclear phosphatase Wip1 in neutrophils could be a new therapeutic strategy in the treatment of several diseases that trigger the inflammatory response and netotic type of death.

The reported study was funded by La Ligue contre le Cancer and RSF (grant \# 19-75-20128).

doi: http://dx.doi.org/10.7124/bc.000A02

\section{V-1. Organization of zebra finch oocyte nucleus}

V. Volodkina $^{1}$, A. Saifitdinova ${ }^{1,2}$, M. Kulak, E. Koshel ${ }^{3}$, S. Galkina ${ }^{1}$, E. Gaginskaya ${ }^{1}$

${ }^{1}$ Saint Petersburg State University, Saint Petersburg, Russia; ${ }^{2}$ Herzen State Pedagogical University of Russia, Saint Petersburg, Russia; ${ }^{3}$ ITMO University, Saint Petersburg, Russia leravolo94@gmail.com

Transcriptionally active lampbrush chromosomes and numerous of different intranuclear structures make nuclei of birds' growing oocytes a working model for investigation of nuclear structure and function. Studies of nucleus content were already made for chicken (Gallus gallus), quail (Coturnix japonica), chaffinch (Fringilla coelebs) and rock pigeon (Columbia livia). Here, we examine the organization of zebra finch (Taeniopygia guttata) oocyte nucleus and look at intranuclear structures. Zebra finch is a singing bird of Estrildidae family that belongs to Passeriformes. This bird is a wellknown model object of neurobiology. To implement our idea we combine the methods of nuclei isolation, confocal microscopy and immunocytochemistry. We found out that there are a lot of ( $\sim 40)$ small bodies (less than $0,5 \mu \mathrm{m}$ ) on the 
nucleus periphery. Intranuclear bodies of $5 \mu \mathrm{m}$ in size contain RNA (RiboGreen), coilin (R288) and FLASH. They are attached to chromosomes. The presence of coilin suggests these structures are some kind of Cajal bodies. However FLASH protein is a marker of histone locus bodies. At the same time zebra finch oocyte nucleus do not contain 'protein bodies' which are common for chaffinch. The lampbrush chromosomes lie in the center of nucleus and do not contact inner nuclear membrane. The most peculiar one is germline-restricted chromosome (GRC) which is the biggest chromosome in karyotype. It is actively transcribed but presents only in gametes. Lampbrush form of this chromosome have obvious condensed 'belts'. There are small coilin positive bodies attached to this 'belts'. We see, that even members of the same order but different families have various intranuclear structures.

Research Resource Centre "Chromas" of Saint Petersburg State University.

doi: http://dx.doi.org/10.7124/bc.000A03

\section{W-1. ROS-induced PML nuclear bodies biogenesis}

Chengchen WU, Pierre Bercier, Shirine Benhenda, Valérie Lallemand-Breitenbach, Hugues de The

College de France U1050, INSERM, CNRS

chengchen.wu@college-de-france.fr

Promyelocytic leukemia nuclear bodies (PML-NBs) are stress-sensitive membrane-less organelles organized by PML, a tumor suppressor protein. PML-NBs are involved in a wide array of biological processes, such as apoptosis, senescence, DNA repair, epigenetic control, control of oncogenesis. The two-step PML-NBs assembly consists first in PML scaffold polymerization in shell-like bodies, then interaction with huge diversity of unrelated proteins recruited in the inner-core. PML-NBs are considered as privileged sites for post-translational modifications (PTM), especially sumoylation. The oxidative stress like that induced by arsenic promotes PML intermolecular disulfide bonds formation and sumoylation, but the mechanism of PML-NBs assembly and dynamics upon stress or at basal level is still poorly understood. Our aim is to unravel the mechanism of PMLNBs biogenesis, assembly and function through the interdisciplinary approach combing molecular and cell biology perspective, and biophysical insight of PML dynamics in living cells to shed light on the way in which PML biological activity modulates the NBs organization. In living cells, we observed PML NBs fusion and fission events. Quantitative analyses of PML dynamics show that PML behaves as a viscous fluid. As2O3 treatment dramatically decreases the diffused PML in nucleus with time, and the dynamic turnover of PML at NBs (FRAP) is abolished. These data revealed that PML has properties at the frontier of liquid and gel, which is strongly modulated by oxidative stress. Upon oxidative stress, PML is covalently bound through intermolecular disulfide bonds, which are formed by the oxidation of cysteines in PML. We show that cysteine mutants in the conserved RBCC domain of PML B2 box are not able to form normal PML-NBs and further sumoylation. These mutants have more diffused PML distributed in the nucleus, but no difference can be observed after $\mathrm{As} 2 \mathrm{O} 3$ 